MATHEMATICS OF COMPUTATION

Volume 71, Number 238, Pages 873-881

S 0025-5718(01)01372-2

Article electronically published on November 21, 2001

\title{
KIRKMAN TRIPLE SYSTEMS OF ORDER 21 WITH NONTRIVIAL AUTOMORPHISM GROUP
}

MYRA B. COHEN, CHARLES J. COLBOURn, LEE A. IVES, AND ALAN C. H. LiNG

\begin{abstract}
There are 50,024 Kirkman triple systems of order 21 admitting an automorphism of order 2 . There are 13,280 Kirkman triple systems of order 21 admitting an automorphism of order 3. Together with the 192 known systems and some simple exchange operations, this leads to a collection of 63,745 nonisomorphic Kirkman triple systems of order 21. This includes all KTS(21)s having a nontrivial automorphism group. None of these is doubly resolvable. Four are quadrilateral-free, providing the first examples of such a $\operatorname{KTS}(21)$.
\end{abstract}

\section{INTRODUCTION}

A Steiner triple system of order $v$, denoted $\operatorname{STS}(v)$, is a pair $(V, \mathcal{B})$, where $V$ is a set of $v$ elements, and $\mathcal{B}$ is a set of 3 -element subsets of $V$ called triples or blocks, so that every 2 -element subset of $V$ occurs in precisely one triple of $\mathcal{B}$. Steiner triple systems have been extensively investigated; see [3].

A parallel class in an $\operatorname{STS}(v)(V, \mathcal{B})$ is a set of disjoint triples whose union is the set $V$; a parallel class therefore contains $v / 3$ triples, and hence an $\operatorname{STS}(v)$ having a parallel class can exist only when $v \equiv 3(\bmod 6)$. When the entire block set $\mathcal{B}$ can be partitioned into parallel classes, such a partition $\mathcal{R}$ is called a resolution of the STS, and the STS is resolvable. If $(V, \mathcal{B})$ is an $\operatorname{STS}(v)$ and $\mathcal{R}$ is a resolution of it, then $(V, \mathcal{B}, \mathcal{R})$ is a Kirkman triple system, and $(V, \mathcal{B})$ is its underlying STS. The distinction between resolvable STSs and KTSs is that a resolvable STS may underlie many nonisomorphic KTSs, since in a KTS the specific resolution is given.

If $(V, \mathcal{B})$ and $(X, \mathcal{D})$ are STSs, an isomorphism from $(V, \mathcal{B})$ to $(X, \mathcal{D})$ is a one-toone mapping $\pi$ from $V$ to $X$ for which $\{x, y, z\} \in \mathcal{B}$ if and only if $\{\pi(x), \pi(y), \pi(z)\} \in$ $\mathcal{D}$. The systems are isomorphic if there is at least one isomorphism from one to the other, and nonisomorphic otherwise. Extending this to Kirkman triple systems, we require an isomorphism to preserve parallel classes, i.e., to map all triples of a parallel class of the first system to triples of a parallel class of the second. An automorphism is an isomorphism from a system to itself. The set of automorphisms forms a group under composition, the automorphism group of the system. The order of the automorphism group is the number of automorphisms which it contains, while the order of an automorphism is the smallest positive number of times that it can be applied in order to obtain the identity map.

Received by the editor May 30, 2000 and, in revised form, August 14, 2000.

2000 Mathematics Subject Classification. Primary 05B07.

Key words and phrases. Kirkman triple system, doubly resolvable design, Steiner triple system, constructive enumeration. 
A parallel class contains $v / 3$ triples, and hence a resolution $\mathcal{R}$ consists of $r=$ $(v-1) / 2$ parallel classes, $\mathcal{R}=\left\{R_{1}, \ldots, R_{r}\right\}$. A parallel class $T$ is orthogonal to the resolution $\mathcal{R}$ if $T \cap R_{i}$ contains zero or one triple for each $1 \leq i \leq r$. Let $\mathcal{R}=\left\{R_{1}, \ldots, R_{r}\right\}$ and $\mathcal{T}=\left\{T_{1}, \ldots, T_{r}\right\}$ be resolutions of the same STS. These two resolutions are orthogonal if the number of triples in $R_{i} \cap T_{j}$ is either zero or one for all $1 \leq i, j \leq r$. When a system has two orthogonal resolutions, it is doubly resolvable.

Kirkman [6] first asked about the existence of Kirkman triple systems in 1850 and solved the case when $v=15$ (the Kirkman 15-schoolgirl problem). Ray-Chaudhuri and Wilson [10] published the first solution to the existence question for KTSs for all $v \equiv 3(\bmod 6)$.

There is a unique $\operatorname{STS}(9)$ up to isomorphism, and it is resolvable. Indeed, it underlies a unique $\operatorname{KTS}(9)$. Of the eighty nonisomorphic STS(15)s, four are resolvable; together they underlie seven nonisomorphic KTS(15)s. The catalogue of seven KTS(15)s was presented by Woolhouse 12, 13, in 1862-63, although the systems themselves were known prior to that time. The KTS(9) and seven KTS(15)s do not admit an orthogonal resolution, and so no $\operatorname{STS}(v)$ is doubly resolvable for $v<21$.

The determination of Kirkman triple systems of the next order, $v=21$, has remained far from complete. Mathon, Phelps, and Rosa [7] found all KTS(21)s having an automorphism which contains three disjoint cycles of length 7, and Tonchev [11] determined that there are 5 further $\operatorname{KTS}(21)$ s having an automorphism of order 7 (and having seven elements fixed by the automorphism). Mathon and Rosa [8] established that there are $48 \mathrm{KTS}(21)$ s having an automorphism of order 5. Altogether, using these systems and applying various substitutions, a total of 192 nonisomorphic $\operatorname{KTS}(21)$ s have been generated; see [4] for details. Of these known designs, all have an odd number of automorphisms (group order 1 occurs 56 times, 366 times, 548 times, 7 three times, 9 twelve times, 21 five times, and 63 twice). None is doubly resolvable.

Our interest was primarily to search for a doubly resolvable STS(21). Doubly resolvable $\operatorname{STS}(v)$ s do not exist when $v \in\{9,15\}$ but do exist for all $v \geq 21$ with $v \equiv$ $3(\bmod 6)$ with 23 possible exceptions [2]. The smallest possible exception occurs when $v=21$, so that the smallest known (nontrivial) doubly resolvable $\operatorname{STS}(v)$ has $v=27$. We are also interested in knowing more about the number and possible structure of $\operatorname{KTS}(21) \mathrm{s}$. For example, a problem posed in [1] is the determination of a quadrilateral-free $\mathrm{KTS}(21)$; a quadrilateral or Pasch configuration is a set of four triples on six elements which pairwise intersect in one element each. Although in the following we do not succeed in finding a doubly resolvable STS(21), we have found four quadrilateral-free $\mathrm{KTS}(21) \mathrm{s}$; and perhaps more importantly, we have found a much larger collection of $\operatorname{KTS}(21)$ s than was known previously.

\section{2. $\operatorname{KTS}(21)$ S WITH AN AUTOMORPHISM OF ORDER THREE}

As a consequence of earlier work [7, 8, 11], all $\operatorname{KTS}(21)$ s having an automorphism of prime order greater than 3 are known. We therefore examine $\operatorname{KTS}(21)$ s having an automorphism of order 3 . Let $\pi$ be an automorphism of a $\operatorname{KTS}(21)(V, \mathcal{B}, \mathcal{R})$. Suppose that $\pi$ has order 3. Let us first consider the action of $\pi$ on the elements in $V$. The elements are partitioned into orbits, where each orbit contains 1 or 3 elements; an element in an orbit of size 1 is fixed by $\pi$. It is easily verified that if $W \subseteq V$ contains precisely the fixed elements under $\pi$, then the triples appearing 
on $W$ form a sub-STS $(|W|)$. It follows that the number of fixed elements must be 0,3 , or 9 . The latter possibility is excluded as follows. A parallel class containing no triple of the sub-KTS(9) contains triples which have at least twice as many elements of $V \backslash W$ as of $W$; but $12(=21-9)$ is not twice as large as 9 , so no such parallel class can be present. Hence the number of fixed elements $e$ cannot be 9 , and $e \in\{0,3\}$.

Next consider the action of $\pi$ on the parallel classes. The ten parallel classes fall into orbits of size 1 or 3 . It follows that the number $c$ of fixed parallel classes is $1,4,7$, or 10. The latter possibility is excluded as follows. If $R$ is a fixed parallel class, then it must contain a fixed triple; but (as we shall see) the number of fixed triples is at most 7 . Hence $c \in\{1,4,7\}$.

Finally let us consider the action on triples. If a triple $T=\{x, y, z\}$ is fixed by $\pi$, then one of two cases arises. In the first, $T$ contains only fixed elements. In the second, we find that $\pi(x)=y, \pi(y)=z$, and $\pi(z)=x$. In either case, an element can be in at most one fixed triple, and hence the number of fixed triples is at most 7. Now the 70 triples of the $\operatorname{KTS}(21)$ are either fixed or in orbits of three triples each, and hence the number $b$ of fixed triples satisfies $b \in\{1,4,7\}$. Further, we have that $c \leq b$ since every fixed parallel class contains a fixed triple.

The class of a $\operatorname{KTS}(21)$ with an automorphism of order 3 is the triple $(e, c, b)$, where $e, c$, and $b$ represent the numbers of fixed elements, fixed parallel classes, and fixed blocks, respectively, under the automorphism of order 3. Our arguments until this point establish that the classes that might arise are $(e, c, b)$ for $e \in\{0,3\}$, $c, b \in\{1,4,7\}$, and $c \leq b$. We can eliminate further cases. In a fixed parallel class, any triple containing a fixed element must be a fixed triple; otherwise, the fixed element appears in more than one triple of the parallel class. Hence when $e=3$, we must have $c=1$ since the only fixed triple containing a fixed element is that triple which contains all three fixed elements.

The nine possibilities for $(e, c, b)$ are listed in Table 1 . For each, the number of $\mathrm{KTS}(21) \mathrm{s}$ in this class is listed, along with the automorphism group orders of the corresponding $\operatorname{KTS}(21)$ s. The totals given in the last line account for nonisomorphic $\operatorname{KTS}(21) \mathrm{s}$.

The computational techniques used to find these systems employ backtracking to find tactical decompositions and nauty to eliminate isomorphic copies among them. Then backtracking is again used to fill (or sign) the tactical decompositions, and nauty used to select one representative of each isomorphism class of the KTS(21)s so produced. For a discussion of backtracking and tactical decompositions, see [5] and references therein; for nauty, see [9].

A single $\operatorname{KTS}(21)$ can admit more than one automorphism of order 3. When this occurs, the KTS has an automorphism group of order divisible by 9 . In such cases, the $\mathrm{KTS}(21) \mathrm{s}$ found can appear in more than one of the classes. In Table 2 sets of classes are given for which at least one $\operatorname{KTS}(21)$ appears in precisely this set of classes. For each set of classes and each automorphism group order, the corresponding number of nonisomorphic $\operatorname{KTS}(21)$ s is given.

We examined all of the $\mathrm{KTS}(21) \mathrm{s}$ and found that four of them are quadrilateralfree. Table 3 gives one example.

Of the $13,280 \operatorname{KTS}(21) \mathrm{s}$ with an automorphism of order 3 , only 11 have the property that every triple belongs to at least one parallel class which is orthogonal to the ten parallel classes of the $\operatorname{KTS}(21)$. This is a basic necessary condition for the design to be doubly resolvable; however, none of the 11 admits an orthogonal 
TABle 1. Numbers of KTS(21)s

\begin{tabular}{|l|rrrrrr|r|}
\hline & \multicolumn{7}{|c|}{ Group Order } \\
Class & 3 & 6 & 9 & 21 & 27 & 63 & Total \\
\hline$(0,1,1)$ & 1004 & 17 & 11 & & & 1 & 1033 \\
$(0,1,4)$ & 20 & 2 & 9 & & & 1 & 32 \\
$(0,1,7)$ & & 1 & 4 & & & & 5 \\
$(0,4,4)$ & 840 & 34 & 20 & 1 & 4 & 1 & 900 \\
$(0,4,7)$ & 20 & & 23 & & & 1 & 44 \\
$(0,7,7)$ & 55 & 4 & 24 & & & 2 & 85 \\
$(3,1,1)$ & 8910 & 100 & 16 & & & & 9026 \\
$(3,1,4)$ & 2170 & 31 & 20 & 4 & 4 & 2 & 2231 \\
$(3,1,7)$ & 16 & & 16 & & 4 & & 36 \\
\hline Total & 13035 & 189 & 45 & 5 & 4 & 2 & 13280 \\
\hline
\end{tabular}

TABLE 2. Types of KTS(21)s

\begin{tabular}{|r|r|l|}
\hline number & group & Automorphism classes \\
\hline 5 & 9 & $(0,1,1)(0,4,4)(0,7,7)(3,1,7)$ \\
1 & 63 & $(0,1,1)(0,4,7)(0,7,7)(3,1,4)$ \\
6 & 9 & $(0,1,1)(0,4,7)(0,7,7)(3,1,4)$ \\
1 & 63 & $(0,1,4)(0,4,4)(0,7,7)(3,1,4)$ \\
1 & 9 & $(0,1,4)(0,4,4)(0,7,7)(3,1,4)$ \\
8 & 9 & $(0,1,4)(0,4,7)(0,7,7)(3,1,1)$ \\
4 & 9 & $(0,1,7)(0,4,4)(0,7,7)(3,1,1)$ \\
4 & 9 & $(0,4,4)(0,4,7)(3,1,1)$ \\
2 & 9 & $(0,4,4)(0,4,7)(3,1,4)$ \\
1 & 9 & $(0,4,4)(3,1,7)$ \\
3 & 9 & $(0,4,4)(0,4,7)(3,1,4)$ \\
4 & 27 & $(0,4,4)(3,1,4)(3,1,7)$ \\
7 & 9 & $(3,1,4)(3,1,7)$ \\
\hline
\end{tabular}

TABLE 3. A quadrilateral-free $\operatorname{KTS}(21)$

\begin{tabular}{|c|c|c|c|c|}
\hline 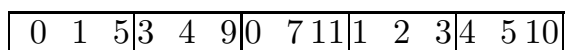 & $\begin{array}{|lll|}1 & 8 & 9\end{array}$ & \begin{tabular}{|lll|lll|}
2 & 0 & 4 & 5 & 3 & 11 \\
\end{tabular} & \begin{tabular}{lll|}
2 & 6 & 10
\end{tabular} & 181920 \\
\hline 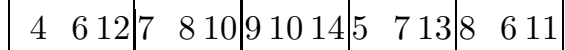 & 101112 & \begin{tabular}{lll|lll}
3 & 8 & 14 & 6 & 7 & 9
\end{tabular} & 119 & 0 \\
\hline 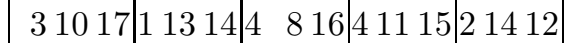 & 61 & $9 \begin{array}{lllllll}9 & 12 & 13\end{array}$ & $\begin{array}{lll}3 & 7 & 15\end{array}$ & \\
\hline 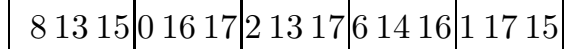 & 01415 & 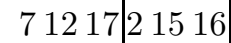 & 11216 & 25 \\
\hline 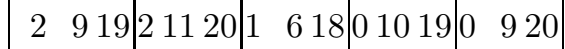 & $2 \quad 7 \quad 18$ & $11119 \mid 11020$ & 818 & 91215 \\
\hline \begin{tabular}{ll|l|l|l|l|l|l|}
71420 & 51218 & 31219 & 812 & 20 & 3 & 13 & 18
\end{tabular} & 41319 & $61320 \mid 41418$ & 51419 & 01316 \\
\hline $111618|61519| 51520|91718| 71$ & 31620 & 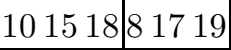 & 417 & 11 \\
\hline
\end{tabular}

resolution. The number of parallel classes orthogonal to the given resolution ranges from 17 to 32 in this set of $11 \mathrm{KTS}(21)$ s; examples of seven disjoint parallel classes orthogonal to the given resolution are present. The numbers of parallel classes of the 13,280 KTS(21)s which are orthogonal to the given resolution appear in Table 4 in parentheses, the numbers are given for the eleven 'candidate' systems. 
TABLE 4. Numbers of parallel classes

\begin{tabular}{|c|rrrrrrrrr|}
\hline Orthogonal p.c.'s & 0 & 1 & 2 & 3 & 4 & 5 & 6 & 7 & 8 \\
\# KTS(21)s & 4088 & 4021 & 1516 & 1388 & 1091 & 431 & 256 & 167 & 122 \\
\hline Orthogonal p.c.'s & 9 & 10 & 11 & 12 & 13 & 14 & 15 & 16 & 17 \\
\# KTS(21)s & 47 & 37 & 47 & 4 & 7 & 12 & 5 & 1 & $7(2)$ \\
\hline Orthogonal p.c.'s & 18 & 20 & 21 & 23 & 25 & 26 & 27 & 32 & 53 \\
\# KTS(21)s & 3 & $8(4)$ & 2 & $7(2)$ & 2 & 2 & $1(1)$ & $7(2)$ & 1 \\
\hline
\end{tabular}

TABLE 5. Underlying STS(21)s

\begin{tabular}{|rr|rr|rr|}
\hline \# STSs & \# KTSs & \# STSs & \# KTSs & \# STSs & \# KTSs \\
\hline 1 & 16 & 1 & 10 & 4 & 8 \\
2 & 7 & 5 & 6 & 3 & 5 \\
65 & 4 & 109 & 3 & 1336 & 2 \\
9904 & 1 & & & & \\
\hline
\end{tabular}

TABLE 6. Group orders for resolvable STS(21)s

\begin{tabular}{|rr|rr|rr|}
\hline Number & Group Order & Number & Group Order & Number & Group Order \\
\hline 1 & $2^{4} \cdot 3^{2} \cdot 7=1008$ & 1 & $2 \cdot 3^{2} \cdot 7^{2}=882$ & 1 & $2 \cdot 3 \cdot 7^{2}=294$ \\
1 & $2 \cdot 3^{2} \cdot 7=126$ & 1 & $2^{3} \cdot 3^{2}=72$ & 4 & $2 \cdot 3 \cdot 7=42$ \\
1 & $3 \cdot 3=27$ & 4 & $2^{3} \cdot 3=24$ & 1 & $3 \cdot 7=21$ \\
5 & $2 \cdot 3^{2}=18$ & 11 & $3^{2}=9$ & 109 & $2 \cdot 3=6$ \\
11290 & 3 & & & & \\
\hline
\end{tabular}

A Steiner triple system of order 21 can underlie numerous KTS(21)s. Among the $13,280 \mathrm{KTS}(21)$ s, we find 11,430 nonisomorphic underlying STS(21)s. Each of these underlies from 1 to 16 nonisomorphic $\operatorname{KTS}(21)$ s among those with an automorphism of order 3; in Table 5 , the column '\#KTSs' gives the number of KTS(21)s underlain, and the column '\#STSs' gives the number of nonisomorphic STS(21)s underlying the given number of KTSs.

The 11,430 underlying STSs can, in general, have larger automorphism groups than do the KTSs which they underlie. Table 6 gives the automorphism group orders for the resolvable STS(21)s.

\section{KTS(21)S WITH AN AUTOMORPHISM OF ORDER TWO}

Let $\pi$ be an automorphism of order 2 acting on a $\operatorname{KTS}(21)$. Evidently $\pi$ fixes at least one element. Since the fixed elements must carry a subsystem, $\pi$ must fix 1 , 3,7 , or 9 elements. Since $\pi$ has order two, any fixed triple either lies entirely on the fixed elements or is of the form $\{f, x, \pi(x)\}$ with $f$ fixed and $x$ not fixed by $\pi$. Now if 7 elements were fixed by $\pi$, they carry an $\operatorname{STS}(7)$, which does not contain two disjoint triples. It follows that at least 7 parallel classes are fixed and that at least 35 triples are fixed. There are not enough fixed triples to achieve this, so $\pi$ cannot fix 7 elements. If $\pi$ were to fix nine elements, there are 12 fixed triples on the fixed elements, and 6 other fixed triples. Each fixed parallel class contains 3 of the 12 and none of the 6 ; or 2 of the 12 and 3 of the 6 ; or 1 of the 12 and all of the 
6. Therefore, there is no integral way to allocate the fixed triples to fixed parallel classes, and hence $\pi$ cannot fix 9 elements.

In addition, $\pi$ cannot fix only one element, since it would then be a reversal, and no reverse $\operatorname{STS}(21)$ exists (see, e.g., [3]). Hence $\pi$ must fix exactly three elements.

It follows that $\pi$ fixes exactly ten triples; one is on the three fixed elements, and nine are of the form $\{f, x, \pi(x)\}$. To determine the number of fixed parallel classes, we proceed as follows. One parallel class contains the fixed triple on the three fixed elements, and hence the class is fixed. Moreover, it contains no further fixed triples as all such triples contain a fixed element. There remain nine fixed triples. When any one is included in a parallel class, the class is fixed under $\pi$. But then the remaining two fixed elements must also appear in fixed triples, and hence the parallel class must contain three such. Hence there are three parallel classes which are fixed, each containing 3 of the 9 remaining fixed triples. The final six parallel classes contain no fixed triples, and indeed are paired under the action of $\pi$.

Summarizing, $\pi$ fixes three elements, ten triples, and four parallel classes; all other elements, triples, and classes are paired by $\pi$. We generated $\operatorname{KTS}(21)$ s with such an automorphism $\pi$ in a number of steps. We outline the process here. We chose $\left(\mathbb{Z}_{9} \times\{0,1\}\right) \cup\left\{\infty_{0}, \infty_{1}, \infty_{2}\right\}$ as the set of elements, with the automorphism $\pi:(x, i) \mapsto(x, 1-i)$ for $(x, i) \in \mathbb{Z}_{9} \times\{0,1\}$ and $\pi$ fixing $\infty_{0}, \infty_{1}, \infty_{2}$.

Generating such a system directly appears to be quite time-consuming, and hence we adopted a number of simplifications of the structure to generate solutions using sequence of backtrack programs, eliminating isomorphic intermediate solutions after each. Each backtrack was implemented in at least two separate ways. Every $\operatorname{KTS}(21)$ admitting $\pi$ can be projected onto $\mathbb{Z}_{9}$ as follows. First, for each of the 60 triples which are paired under $\pi$, we delete one triple from the pair; when they appear in paired parallel classes, we select triples to remove so that one of the parallel classes in each pair has all of its triples removed. We then project onto $\mathbb{Z}_{9}$ by mapping $(x, 0)$ and $(x, 1)$ both to $x$ for each $x \in \mathbb{Z}_{9}$. We then delete the infinite elements. The resulting collection of blocks consists of triples, pairs, and singletons on the elements of $\mathbb{Z}_{9}$ (one fixed triple has been deleted, and the remaining nine reduced to singletons). This 'design' has the property that every 2 -subset of $\mathbb{Z}_{9}$ occurs in exactly two blocks, since in the $\operatorname{KTS}(21)$ there are four 2-subsets of the form $\{(x, i),(y, j)\}$ each occurring once, but these arise in two paired 2-subsets. In addition, the projected KTS has its blocks partitioned into seven classes, as follows:

1. one parallel class of three triples;

2. three parallel classes, each containing three singletons and two triples; and

3. three sets, each containing four triples and three pairs so that every element is in exactly two.

In addition, the nine singletons are all distinct. If one further omits this information about partitioning the blocks, the set system of interest has nine elements and thirty blocks of which 21 are triples and 9 are pairs; the singletons can be omitted. The basic properties are that every 2 -subset occurs in exactly two blocks, and every element occurs in exactly two pairs. We employed backtracking and nauty to establish that there are precisely 8,716 nonisomorphic set systems.

Next we determined using backtracking all of the partitions of the set systems into the seven classes detailed above. We observed that a repeated triple cannot occur in a system which is a projection of a KTS, and further that in the last three classes, no two blocks can intersect in two elements. This restricted the number 
TABle 7. Parallel classes in 50,024 KTSs

\begin{tabular}{|c|rrrrrrrrr|}
\hline Orthogonal p.c.'s & 0 & 1 & 2 & 3 & 4 & 5 & 6 & 7 & 8 \\
\# KTS(21)s & 12528 & 11955 & 10996 & 7003 & 3954 & 2035 & 860 & 397 & 165 \\
\hline Orthogonal p.c.'s & 9 & 10 & 11 & 12 & 13 & 15 & 18 & 20 & 23 \\
\# KTS(21)s & 73 & 32 & 11 & 5 & 4 & 2 & 1 & 2 & 1 \\
\hline
\end{tabular}

TABLE 8. Underlying STS(21)s

\begin{tabular}{|rr|rr|rr|}
\hline \# STSs & \# KTSs & \# STSs & \# KTSs & \# STSs & \# KTSs \\
\hline 3 & 11 & 1 & 10 & 1 & 9 \\
13 & 8 & 5 & 7 & 25 & 6 \\
61 & 5 & 312 & 4 & 758 & 3 \\
6814 & 2 & 32228 & 1 & & \\
\hline
\end{tabular}

of solutions without removing any of the eventual KTSs to be produced. Together with the seven class partition, we found exactly 788,813 projected KTSs which are nonisomorphic.

Again with backtracking, we determined how each pair and singleton could be extended to a triple by adding one of $\infty_{0}, \infty_{1}$, and $\infty_{2}$. Since each fixed element must appear with each of the different elements from $\mathbb{Z}_{9}$, this selection of fixed elements to include is quite restrictive. Hence we found that the number of solutions in which the fixed elements are re-introduced drops; indeed, the number of nonisomorphic solutions is 174,144 .

The final phase determines, for each element $x$ of $\mathbb{Z}_{9}$ in each block, whether to associate it with $(x, 0)$ or with $(x, 1)$. The constraint is that every orbit of 2-subsets must be represented exactly once. Then applying $\pi$ produces the full $\operatorname{KTS}(21)$. With a final backtrack, we produced exactly 50,024 nonisomorphic KTS(21)s having an automorphism of order 2 .

Of these, 189 have an automorphism group of order 6 and all others admit no automorphism except for powers of $\pi$. The number of KTS(21)s with a group of order 6 agrees with the result in Table 1 and so the results of this and the preceding section are consistent.

Once again, none is doubly resolvable. Indeed, each has at least one triple that occurs in no parallel class orthogonal to the given resolution, so each is in one sense far from doubly resolvable. Table 7 gives the possible numbers of parallel classes which are orthogonal to the given resolution, along with the number of KTSs for which this number arises.

There are 40,221 nonisomorphic STS(21)s underlying the 50,024 systems. The number of $\operatorname{KTS}(21)$ s that each underlies is given in Table 8, Of the 40,221 underlying STS(21)s, there are 40,115 with a group of order $2 ; 8$ with a group of order 4 ; and 98 with a group of order 6 .

\section{4. $\operatorname{KTS}(21) \mathrm{S}$ BY EXCHANGES}

Of the 13,280 systems with an automorphism of order 3 , exactly 85 already appear among the $192 \mathrm{KTS}(21) \mathrm{s}$ in [4]. None of the 50,024 KTS(21)s having an automorphism of order 2 appear among the 192 known systems. We produced 
TABle 9. KTS(21) Summary

\begin{tabular}{|r|r|r|r|r|r|}
\hline Group Order & {$[4]$} & Exchange & $\Phi \underline{2}$ & $\$ 3$ & Total \\
\hline 1 & 56 & 523 & & & 579 \\
2 & & & & 49835 & 49835 \\
3 & 66 & & 13035 & & 13035 \\
5 & 48 & & & & 48 \\
6 & & & 189 & 189 & 189 \\
7 & 3 & & & & 3 \\
9 & 12 & & 45 & & 45 \\
21 & 5 & & 5 & & 5 \\
27 & & & 4 & & 4 \\
63 & 2 & & 2 & & 2 \\
\hline total & 192 & 523 & 13280 & 50024 & 63745 \\
\hline
\end{tabular}

further $\operatorname{KTS}(21) \mathrm{s}$ as well as follows. Consider a $\operatorname{KTS}(21)$ containing two parallel classes $P$ and $Q$ for which $P \cup Q$ is disconnected. Then one component must contain three blocks each of $P$ and $Q$, and the other component four blocks from each. Let $C$ contain the six blocks of the smaller component. Then we can exchange to find a new $\operatorname{KTS}(21)$ by replacing the parallel classes $P$ and $Q$ by the parallel classes $(P \backslash C) \cup(Q \cap C)$ and $(P \cap C) \cup(Q \backslash C)$. The resulting $\operatorname{KTS}(21)$ has the same underlying $\operatorname{STS}(21)$ as the original, but need not have the same automorphism group.

We found all pairs of parallel classes in the 63,222 KTS(21)s including the 192 known systems, and those with automorphisms of order 2 or 3 . We applied this exchange procedure to produce all possible $\mathrm{KTS}(21) \mathrm{s}$ that result from some sequence of such exchanges: in total, 523 nonisomorphic $\operatorname{KTS}(21)$ s that are not among those generated earlier. None is doubly resolvable, and none is quadrilateral-free. In Table 9, we summarize the numbers of $\operatorname{KTS}(21)$ s known from [4, 43, 42, and obtained by exchanges, along with a total which accounts for overlaps among these collections. The web page http://www.public.asu.edu/ ccolbou/src/kts21.txt gives a list of the 63,745 nonisomorphic systems now known.

\section{ACKNOWLEDGMENTS}

Thanks to Alex Rosa and Rudi Mathon for information about the 192 KTS(21)s. Research of the authors is supported by ARO grant DAAG55-98-1-0272 (Colbourn).

\section{REFERENCES}

1. Y.M. Chee, C.J. Colbourn, and A.C.H. Ling, Asymptotically optimal erasure-resilient codes for large disk arrays, Discrete Appl. Math. 102 (2000), 3-36. MR 2001i:68038

2. C.J. Colbourn, E.R. Lamken, A.C.H. Ling, and W.H. Mills, The existence of Kirkman squares - doubly resolvable $(v, 3,1)$-BIBDs, Designs Codes Crypt., to appear.

3. C.J. Colbourn and A. Rosa, Triple Systems, Oxford University Press, 1999.

4. I.J. Dejter, F. Franek, and A. Rosa, A completion conjecture for Kirkman triple systems, Utilitas Math. 50 (1996), 97-102. MR 97i:05013

5. P.B. Gibbons, Computational methods in design theory, in CRC Handbook of Combinatorial Designs, (C.J. Colbourn and J.H. Dinitz, editors), CRC Press, Boca Raton FL, 1996, pp. 718-740.

6. T.P. Kirkman, Note on an unanswered prize question, Cambridge and Dublin Math. J. 5 (1850), 255-262. 
7. R. Mathon, K.T. Phelps, and A. Rosa, A class of Steiner triple systems of order 21 and associated Kirkman triple systems, Math. Comput. 37 (1981), 209-222. MR 82k:05022

8. R. Mathon and A. Rosa, The 4-rotational Steiner and Kirkman triple systems of order 21, Ars Combinat. 17 (1984), 241-250. MR 85c:05028

9. B. D. McKay, Nauty Users Guide (Version 1.5), Technical Report TR-CS-90-02, 1990 Computer Science Department, Australian National University.

10. D.K. Ray-Chaudhuri and R.M. Wilson, Solution of Kirkman's schoolgirl problem, Proc. Sympos. Pure Math. 19, American Math. Society, Providence RI (1971), pp. 187-203. MR 47:3195

11. V.D. Tonchev, Steiner triple systems of order 21 with automorphisms of order 7, Ars Combinat. 23 (1987), 93-96. MR 88d:05028

12. W.S.B. Woolhouse, On triadic combinations of 15 symbols, Lady's and Gentleman's Diary (1862) 84-88. Reprinted in Assurance Magazine 10, 275-281.

13. W.S.B. Woolhouse, On triadic combinations, Lady's and Gentleman's Diary (1863), 79-90.

Department of Computer Science, University of Auckland, Auckland, New Zealand

E-mail address: myra@cs.auckland.ac.nz

Department of Computer Science and Engineering, Arizona State University, Tempe, ARIZONA 85287-5406

E-mail address: Charles.Colbourn@asu.edu

Department of Mathematics and Statistics, University of Vermont, Burlington, VerMONT 05405

Department of Computer Science, University of Vermont, Burlington, Vermont 05405

E-mail address: aling@emba.uvm.edu 